\title{
REMOVING POINT SINGULARITIES OF RIEMANNIAN MANIFOLDS
}

\author{
P. D. SMITH AND DEANE YANG
}

\begin{abstract}
We study the behavior of geodesics passing through a point singularity of a Riemannian manifold. In particular, we show that if the curvature does not blow up too rapidly near the singularity, then the singularity is at worst an orbifold singularity. The idea is to construct the exponential map centered at a singularity. Since there is no tangent space at the singularity, a surrogate is needed. We show that the vector space of radially parallel vector fields is well defined and that there is a correspondence between unit radially parallel vector fields and geodesics emanating from the singular point.
\end{abstract}

\section{INTRODUCTION}

We wish to study the following question: given a point singularity (which will be defined more precisely later) of a Riemannian manifold, when can both the manifold structure and the Riemannian metric extended smoothly across the singularity? If the extended metric is to be a reasonably nice one, say a $C^{2}$-metric, then the geodesics passing through the singularity must behave well. This observation will be the crux of our analysis of a point singularity. We will start with a qualitative assumption about the geodesics, namely that there are no arbitrarily small geodesic loops passing through the singularity. Combining this with suitable assumptions on the Riemannian manifold and its curvature, we will show that the geodesics passing through the singularity behave as expected and that an exponential map centered there is well defined. Using exponential (i.e., geodesic normal) coordinates, the metric can be extended continuously across the singularity. To obtain better regularity of the metric, we then use Jost-Karcher's construction of almost linear and harmonic coordinates.

It seems to us that the assumption of no arbitrarily short geodesic loops should not be needed and should follow from the other assumptions. However, without an exponential map centered at the singularity, we have been unable to see how to eliminate it from the theorem.

\section{Preliminaries}

Let $\widetilde{M}$ be an $n$-dimensional smooth manifold with a $C^{1}$ Riemannian metric $\tilde{g}$. The metric $\tilde{g}$ induces a distance function

$$
d(x, y)=\inf _{\gamma} l[\gamma], \quad x, y \in \widetilde{M},
$$

Received by the editors March 15, 1989 and, in revised form, May 9, 1990.

1980 Mathematics Subject Classification (1985 Revision). Primary 53C20. 
where the infimum is taken over all smooth curves with endpoints at $x$ and $y$ and $l[\gamma]$ denotes the length of the curve $\gamma$. The function $d(x, y)$, in turn, defines a metric space structure on $\widetilde{M}$. Let $M$ denote the completion of $\widetilde{M}$ as a metric space.

We say $p \in M$ is a point singularity of $\widetilde{M}$ if there exists a neighborhood $N \subset$ $M$ of $p$ such that $N \cap \widetilde{M}=N \backslash\{p\}$. The simplest example of a nonremovable point singularity is the origin in $\mathbf{R}^{3}$, where

$$
\widetilde{M}=\left\{(x, y, z) \in \mathbf{R}^{3} \mid x^{2}+y^{2}-z^{2}=0, z>0\right\} .
$$

Given a point singularity $p \in M$, we say that it is conic if for $x \in \widetilde{M}$ suffciently close to $p$, the distance function $r(x)=d(p, x)$ is $C^{1}$. In particular, this implies that the level sets of $r$ are all difieomorphic to a fixed compact $C^{1}$-manifold $S$ and that for sufficiently small $\varepsilon>0$,

$$
r^{-1}((0, \varepsilon)) \cong(0, \varepsilon) \times S,
$$

and that the matrix $\tilde{g}$ can be written in the form $\tilde{g}=d r^{2}+g_{r}$, where $g_{\rho}$ is the metric induced by $\tilde{g}$ on the level set $r^{-1}(\rho)$.

We will denote the Levi-Civita connection associated with the Riemannian metric by $\nabla$, so that given vector fields $V$ and $W$, the covariant derivative of $V$ with respect to $W$ is the vector field $\nabla_{W} V$.

The Riemannian curvature operator is defined to be

$$
R(X, Y) Z=\left(\nabla_{X} \nabla_{Y}-\nabla_{Y} \nabla_{X}-\nabla_{[X, Y]}\right) Z .
$$

Given $x \in \widetilde{M}$ and a 2-plane $E \subset T_{x} \widetilde{M}$, let $K(x, E)$ denote the sectional curvature of $E$. In other words, given orthonormal vectors $e_{1}, e_{2} \in E$,

$$
K(x, E)=R\left(e_{1}, e_{2}\right) e_{2} \cdot e_{1} .
$$

Also, define

$$
|K|(x)=\sup _{E \subset T_{x} M}|K(x, E)| .
$$

The curvature tensor is apparently well defined only if the metric is $C^{2}$. On the other hand, if we are willing to fix a set of coordinates, it is easily checked that the formula for the components of the Riemann curvature tensor given in terms of the metric and its derivatives up to second order is well defined in the distribution sense if the metric is $C^{1}$. In particular, a $C^{1}$-metric $g$ is said to have continuous Riemann curvature if there exist coordinates such that the components of the Riemann curvature are continuous functions. For convenience the rest of our paper is written as if the metric $\tilde{g}$ is $C^{2}$. On the other hand, we do want to observe here that it suffices to assume that $\tilde{g}$ is $C^{1}$ with continuous curvature. Until near the end of the paper when we construct new coordinates, all of the vector fields and tensors that we use can be thought of as being written with respect to the coordinates in which the curvature is continuous. It is shown in [8] that the existence and uniqueness of geodesics with given initial conditions and the Jacobi equation for variations of geodesics still hold for a $C^{1}$ metric with continuous curvature. Since this is all that we need, all of our arguments still work. Also, all of the arguments used in [10 and 9] to construct almost linear and harmonic coordinates carry through to prove the following (also see [5]): 
Theorem 2.1. Let $g$ be a $C^{1}$ metric with continuous Riemann curvature. Then there exist local (harmonic) coordinates in which the components of the metric tensor are $C^{1, \alpha}$ functions for any $0<\alpha<1$.

\section{STATEMENTS OF MAIN THEOREMS}

Given $\varepsilon>0$ and $p \in M$, a metric space, denote

$$
\widetilde{B}_{\varepsilon}(p)=\{x \in M \mid 0<d(p, x)<\varepsilon\} .
$$

Theorem 3.1. Let $\widetilde{M}$ be an $n$-dimensional manifold, $n \geq 3$, with a $C^{1}$ Riemannian metric $\tilde{g}$ with continuous Riemann curvature. Let $p$ be a point singularity of $\widetilde{M}$.

Assume that there exists $\varepsilon>0$ such that the following are satisfied:

(i) $\widetilde{B}_{\varepsilon}(p)$ is simply connected.

(ii)

$$
\int_{0}^{\varepsilon} s \kappa(x) d s<\infty
$$

where

$$
\kappa(s)=\sup _{s \leq d(p, x) \leq \varepsilon, E \subset T_{x} M}|K(x, E)| .
$$

(iii) There is no geodesic loop in $\widetilde{B}_{\varepsilon}(p)$ with both endpoints at $p$.

Then there exists a smooth manifold structure on a neighborhood of $p$ compatible with the one on $\widetilde{M}$ such that $\tilde{g}$ extends to a $C^{1}$ metric $g$ across $p$. Moreover, if the Ricci curvature on $\widetilde{M}$ satisfies

$$
\|\operatorname{Rc}(\tilde{g})\|_{L^{p}}<\infty, \quad p>n,
$$

then there exist coordinates in which the metric $g$ is $C^{1, \alpha}$, where $0<\alpha<$ $1-n / p$. If, in addition, the Ricci curvature satisfies the following Hölder bound,

$$
\|\operatorname{Rc}(\tilde{g})\|_{C^{k, \alpha}}<\infty, \quad k \geq 0,0<\alpha<1,
$$

then there exist coordinates in which the metric $g$ is $C^{k+2, \alpha}$.

Corollary 3.2. Assume all the hypotheses of Theorem 1, except (i). Then the point $p$ is an orbifold singularity.

Theorem 3.3. Let $\widetilde{M}$ be a 2-dimensional manifold with a $C^{1}$ Riemannian metric $\tilde{g}$ with continuous Riemannian curvature. Let $p$ be a point singularity of $\widetilde{M}$. Using the same definitions as in Theorem 3.1 assume (ii) of Theorem 3.1 and the following.

(i) The closed ball $\bar{B}_{\varepsilon}(p)$ in the metric completion $M$ is compact.

(ii) The Gauss-Bonnet theorem holds on $B_{\varepsilon}(p) \subset M$. In other words, given any domain $D \subset B_{\varepsilon}(p)$ with smooth boundary $\partial D \subset \widetilde{B}_{\varepsilon}(p)$,

$$
\int_{D} K d A+\int_{\partial D} k d s=2 \pi \chi(D),
$$

where $K$ is the Gauss curvature, $k$ is the curvature of the curve $\partial D, \chi(D)$ is the Euler characteristic of $D$.

Then the conclusions of Theorem 2 hold. 


\section{EXISTENCE AND UNIQUENESS OF GEODESIC RAYS}

A curve $\gamma:[0,1] \rightarrow M$ is a geodesic if given any two sufficiently close points on the curve, $\gamma$ is curve of smallest length joining them. When $\gamma$ lies on $\widetilde{M}$, it must then satisfy the Euler-Lagrange equations corresponding of the length functional for curves. We shall, in fact, always assume that the map $\gamma$ is a critical point for the energy functional,

$$
E[\gamma]=\int_{\gamma}\left|\gamma^{\prime}\right|^{2} d s,
$$

so that $\gamma$ satisfies the corresponding Euler-Lagrange equations

$$
\nabla_{\gamma^{\prime}} \gamma^{\prime}=0 \text {. }
$$

In particular, this implies that $\gamma$ is a $C^{2}$ map and $\gamma$ is parameterized by a constant times arclength, where the constant is simply the length of $\gamma$. The curve $\gamma$ will be called a minimal geodesic if

$$
d(\gamma(0), \gamma(1))=l[\gamma] .
$$

Definition. Let $p$ be a point singularity of $M$. A geodesic $\gamma:[0,1] \rightarrow M$ such that $\gamma(0)=p$, will be called a geodesic ray.

The following is a straightfoward application of the Arzela-Ascoli theorem.

Proposition 4.1. Let $p$ be a point singularity of the Riemannian manifold $\widetilde{M}$. Given any $x, y \in \widetilde{M} \cup\{p\}$, there exists a minimal geodesic ray joining $x$ to $y$.

Proof (following [7]). We give a careful proof, since it is slightly different from the usual situation. The standard argument is to take a minimizing sequence of continuous curves and apply the following version of the Arzela-Ascoli theorem (a proof can be found in [6, §VII.5]) to obtain a limiting curve:

Theorem 4.2. Let $M$ be a complete metric space and $N$ a compact metric space. Let

$$
\gamma_{i}: N \rightarrow M, \quad i \in \mathbf{Z}^{+},
$$

be a sequence of continuous maps satisfying the following:

(i) for any $\varepsilon>0$, there exists $\delta>0$ such that for any $i \in \mathbf{Z}^{+}, x, y \in N$ satisfying $d_{N}(x, y)<\delta$,

$$
D_{M}\left(\gamma_{i}(x), \gamma_{i}(y)\right)<\varepsilon .
$$

(ii) For any $x \in N$, the set $\left\{\gamma_{i}(x), i \in \mathbf{Z}^{+}\right\}$is relatively compact in $M$. Then there exists a subsequence of $\left\{\gamma_{i}\right\}$ which is uniformly convergent.

We reduce to $y=p$ as follows: if neither $x$ nor $y$ is $p$, then either $d(x, y)<d(x, p)+d(p, y)$ or $d(x, y)=d(x, p)+d(p, y)$. In the former case, the minimizing geodesic will not pass through $p$. In this case, the standard minimizing argument (which is essentially what is given below) works. In the latter, we construct the minimizing geodesic as the union of minimizing geodesics joining $x$ to $p$ and $p$ to $y$.

Now assume $y=p$. Let $\gamma_{i}:[0,1] \rightarrow M$ define a sequence of continuous curves joining $p$ to $x$ such that each $\gamma_{i}$ is parameterized by a constant 
times arclength and the lengths $l\left[\gamma_{i}\right]$ form a decreasing sequence converging to $d(p, x)$.

By taking a subsequence, if necessary, we can always assume that $l\left[\gamma_{i}\right] \leq$ $2 d(p, x)$. This implies that

$$
d\left(\gamma_{i}(x), \gamma_{i}(t)\right) \leq l\left[\left.\gamma_{i}\right|_{[s, t]}\right]=l\left[\gamma_{i}\right]|s-t| \leq 2 d(p, x)|s-t|,
$$

showing that (i) is satisfied.

Now fix $t \in[0,1]$. All but a finite subset of $\left\{\gamma_{i}(t)\right\}$ lie in a closed (and compact) ball $\bar{B}_{\varepsilon}(x) \subset \widetilde{M}$, for some fixed $\varepsilon<d(p, x)$. This implies (ii). Applying the Arzela-Ascoli theorem, we obtain a subsequence which converges uniformly to a continuous curve $\gamma:[0,1] \rightarrow M$. By the lower semicontinuity of the length function, $l[\gamma]=d(p, x)$. Q.E.D.

\section{ESTIMATES OF JACOBI FIELDS}

The key tool in our proof will be estimates of Jacobi fields defined by families of geodesic rays emanating from the singular point $p$.

Consider a differentiable family of geodesics,

$$
\Gamma:(0,1] \times[-\delta, \delta] \rightarrow \widetilde{M} .
$$

Denote

$$
\frac{\partial}{\partial s}=\frac{\partial \Gamma}{\partial s}, \quad \frac{\partial}{\partial t}=\frac{\partial \Gamma}{\partial t},
$$

where $\Gamma=\Gamma(s, t)$. Since

$$
\nabla_{\partial / \partial s} \frac{\partial}{\partial s}=0 \quad \text { and } \quad \nabla_{\partial / \partial s} \frac{\partial}{\partial t}=\nabla_{\partial / \partial t} \frac{\partial}{\partial s}
$$

the following holds:

$$
\begin{aligned}
\nabla_{\partial / \partial s} \nabla_{\partial / \partial s} \frac{\partial}{\partial t} & =\nabla_{\partial / \partial s} \nabla_{\partial / \partial t} \frac{\partial}{\partial s} \\
& =\nabla_{\partial / \partial t} \nabla_{\partial / \partial s} \frac{\partial}{\partial s}+R\left(\frac{\partial}{\partial s}, \frac{\partial}{\partial t}\right) \frac{\partial}{\partial s} \\
& =R\left(\frac{\partial}{\partial s}, \frac{\partial}{\partial t}\right) \frac{\partial}{\partial s} .
\end{aligned}
$$

Therefore, the vector field $J=\partial / \partial t$, which represents an infinitesimal variation of geodesics, satisfies the differential equation

$$
\nabla_{\partial / \partial s} \nabla_{\partial / \partial s} J+R\left(J, \frac{\partial}{\partial s}\right) \frac{\partial}{\partial s}=0 .
$$

This equation is called the Jacobi equation and given any geodesic $\gamma$, a vector field $J$ along $\gamma$ which solves (5.1) is called a Jacobi field. We shall study Jacobi fields by comparing the sectional curvature to a function $\kappa$ which is allowed to blow up at one of the endpoints of the geodesic.

The general approach taken here is based on that of $\mathrm{H}$. Karcher: we learned it from an exposition of J. Jost, [9] (also, see [11, 10, 3]).

We reduce (5.1) to a linear selfadjoint second order system of ODE's by fixing a geodesic ray $\gamma:(0,1] \rightarrow \widetilde{M}$ and a frame of parallel orthonormal vector 
$e_{1}, \ldots, e_{n}$. Given a vector field $J$ along $\gamma$, we can expand with respect to the frame, obtaining $J=J^{i} e_{i}$. The Jacobi equation then becomes

$$
\left(J^{i}\right)^{\prime}+K_{j}^{i} J^{j}=0, \quad i \leq j \leq n,
$$

where $K_{j}^{i}=R\left(e_{j}, \gamma^{\prime}\right) \gamma^{\prime} \cdot e_{i}=K_{i}^{j}$. Abusing notation slightly, we shall write this last equation in the following form:

$$
J^{\prime \prime}+K J=0,
$$

where $J=\left[J^{i}\right]$ and $K=\left[K_{j}^{i}\right]$ is a continuous, symmetric $n \times n$ matrix-valued function on $(0,1]$.

Throughout this section we will assume that $K(s)$ is continuous on $(0,1]$ and that the following estimate holds:

$$
|K(s) V| \leq \kappa(s)|V|, \quad V \in \mathbf{R}^{n}, s \in(0,1],
$$

where the function $\kappa(s)$ satisfies

$$
\int_{0}^{1} s \kappa(s) d s \leq \delta^{2}<1
$$

Proposition 5.5. Given $V \in \mathbf{R}^{n}$ there exists a unique solution $J \in C^{1}([0,1]) \cap$ $C_{\text {loc }}^{2}((0,1])$ to

$$
J^{\prime \prime}+K J=0, \quad J(0)=0, \quad J^{\prime}(0)=V .
$$

Given $V, V^{\prime} \in \mathbf{R}^{n}$, there exists a unique solution $J \in C^{0}([0,1]) \cap C_{\mathrm{loc}}^{2}((0,1])$ such that

$$
J^{\prime \prime}+K J=0, \quad J(1)=V, \quad J^{\prime}(1)=V^{\prime} .
$$

Moreover, the solution of each initial value problem depends continuously on the initial data.

Proof. Both parts follow easily by applying standard contraction map arguments to the following integral equations: for (5.6), the trick is to solve for $J^{\prime} \in$ $C^{0}([0,1])$ satisfying

$$
J^{\prime}(s)=J^{\prime}(0)-\int_{0}^{s} K(s)\left(\int_{0}^{t} J^{\prime}(\tau) d \tau\right) d t,
$$

and for (5.7), solve for $J \in C^{0}([0,1])$ satisfying

$$
J(s)=J(1)+(s-1) J^{\prime}(1)-\int_{s}^{1}(t-s) K J d t \text {. Q.E.D. }
$$

Applying this theorem, we let $\psi \in C^{1}([0,1])$ be the unique solution to

$$
\psi^{\prime \prime}+\kappa \psi=0,
$$

with $\psi(0)=0, \psi^{\prime}(0)=1$. Observe that as a consequence of (5.4), $\psi$ is an increasing function on $[0,1]$. Let $\tilde{\psi}$ be the unique solution to (8) such that $\tilde{\psi}(1)=0$ and $\tilde{\psi}^{\prime}(1)=-1$. By the Sturm separation theorem, $\tilde{\psi}$ is a positive decreasing function on $[0,1]$.

We also define $\hat{\psi}$ to be the unique solution of

$$
\hat{\psi}^{\prime \prime}-\kappa \hat{\psi}=0, \quad \hat{\psi}(0)=0, \quad \hat{\psi}^{\prime}(0)=1 .
$$


The following is easily verified:

$$
\psi(1) s \leq \psi(s) \leq s \leq \hat{\psi}(s) \leq \hat{\psi}(1) s .
$$

Remark. The examples to keep in mind are the following:

$$
\kappa(s)=\left(\frac{\pi}{2}\right)^{2}, \quad \psi(s)=\sin \frac{\pi}{2} s, \quad \hat{\psi}(s)=\sin \frac{\pi}{2} s
$$

and, given $0<\varepsilon, \alpha<1, c=[\alpha(\alpha+1)]^{-1}$,

$$
\kappa(s)=s^{-2+\alpha}, \quad \psi(s)=s\left(1-c s^{\alpha}\right), \quad \hat{\psi}(s)=s\left(1-\varepsilon+c s^{\alpha}\right) .
$$

The key example of a function which does not satisfy (4) is $\kappa(s)=s^{-2}$.

The geometric interpretation of the functions $\psi$ and $\hat{\psi}$ is that they define rotationally symmetric metrics of the form $d r^{2}+\psi^{2} \Theta$ and $d r^{2}+\hat{\psi}^{2} \Theta$, where $\Theta$ is the standard metric on $S^{n-1}$. These metrics are $C^{1}$ across the origin, have well-behaved geodesics passing through the origin, and have sectional curvatures equal to $\pm \kappa(r)$. They are the model metrics against which the metric $g$ on $\widetilde{M}$ will be compared. In particular, the functions given by (10) corresponding to metrics of constant positive and negative sectional curvature.

We begin with some results that require only an upper bound for sectional curvature. The following is essentially the Sturm comparison theorem:

Lemma 5.11. Let $J(s) \in C^{0}([0,1]) \cap C_{\mathrm{loc}}^{2}((0,1])$, be a solution of (5.2). Then if $J(0)=0$, then the function $|J(s)| / \psi(s)$ is increasing for $0<s<1$. On the other hand, $J(1)=0$, then the function $|J(s)| / \tilde{\psi}(s)$ is decreasing for $0<s<1$. Proof. A straightforward calculation shows that the function $|J|(s)=|J(s)|$ satisfies the following differential inequality:

$$
|J|^{\prime \prime}+\kappa|J| \geq 0 \text {. }
$$

Now consider $|J| / \psi$ on the interval $(0,1]$. Differentiating it, we obtain

$$
\left(\frac{|J|}{\psi}\right)^{\prime}=\frac{|J|^{\prime} \psi-|J| \psi^{\prime}}{\psi^{2}}
$$

It therefore suffices to show that the numerator of the right-hand side is nonnegative for $s \in(0,1]$.

First, observe that (5.12) implies that

$$
\left(|J|^{\prime} \psi-|J| \psi^{\prime}\right)^{\prime}=|J|^{\prime \prime} \psi-|J| \psi^{\prime \prime} \geq 0, \quad s>0 .
$$

Since $|J| \rightarrow 0$ as $s \rightarrow 0$, there must be a sequence $s_{i} \rightarrow 0$ such that $|J|^{\prime}\left(s_{i}\right)>0$. It then follows that for $s \geq s_{i}$,

$$
\left(|J|^{\prime} \psi-|J| \psi^{\prime}\right)(s) \geq\left(|J|^{\prime} \psi-|J| \psi^{\prime}\right)\left(s_{i}\right) \geq-|J| \psi^{\prime}\left(s_{i}\right) .
$$

Letting $i \rightarrow \infty$, we obtain the desired conclusion.

The same argument applies to the case when $J(1)=0$. Q.E.D.

Theorem 5.13. Given $V_{0}, V_{1} \in \mathbf{R}^{n}$, there exists a unique solution $J \in C^{0}([0,1])$ $\cap C_{\mathrm{loc}}^{2}((0,1])$ to the following boundary value problem:

$$
J^{\prime \prime}+K J=0 ; \quad J(0)=V_{0} ; \quad J(1)=V_{1} .
$$

If $V_{0}=0$, then $J \in C^{1}([0,1])$, and the following bounds hold for any $0 \leq s \leq 1$,

$$
|J(s)| \leq \frac{|J(1)|}{\psi(1)} \psi(s), \quad\left|J^{\prime}(s)\right| \leq \frac{|J(1)|}{\psi(1)} \psi^{\prime}(s) .
$$


Proof. Uniqueness of the solution follows directly from Lemma 5.11 with $V=$ 0 .

Let $e_{1}, \ldots, e_{n}$ be the standard basis of $\mathbf{R}^{n}$ and $J_{i}$ the unique solution to (5.2) such that $J_{i}(1)=V_{1}$ and $J_{i}^{\prime}(0)=e_{i}$. By the uniqueness of the boundary value problem, $J_{1}(0), \ldots, J_{n}(0)$ must be linearly independent. Therefore, there is a unique linear combination $J=a^{i} J_{i}$ such that $J(0)=a^{i} J_{i}(0)=V_{0}$.

The bound on $|J(s)|$ follows directly from Lemma 5.11. Also applying Lemma 5.11, we obtain

$$
\begin{aligned}
\left|J^{\prime}(s)\right| & \leq\left|J^{\prime}(0)\right|+\int_{0}^{s} \kappa(t)|J(t)| d t \\
& \leq \frac{|J(1)|}{\psi(1)}\left(1+\int_{0}^{s} \kappa(t) \psi(t) d t\right) \leq \frac{|J(1)|}{\psi(1)} \psi^{\prime}(s) \text {. Q.E.D. }
\end{aligned}
$$

To obtain a bound on $|J(1)|$ in terms of $\left|J^{\prime}(0)\right|$, we need to use the lower bound on the curvature.

Lemma 5.14. Given a solution $J \in C^{1}([0,1])$ to $(5.2)$,

$$
|J(s)| \leq\left|J^{\prime}(0)\right| \hat{\psi}(s) \leq\left|J^{\prime}(0)\right| \hat{\psi}(1) s ; \quad\left|J^{\prime}(s)\right| \leq\left|J^{\prime}(0)\right| \hat{\psi}^{\prime}(s) .
$$

Proof. We follow the proof of Lemma 2.2.4 in [9].

Denote $V=J^{\prime}(0)$ and $A(s)=V s$. Let $b$ be the unique solution to $b^{\prime \prime}=$ $\kappa|J|, b(0)=b^{\prime}(0)=0$. Let $a(s)=|V|(\hat{\psi}(s)-s)$ and observe that $a$ satisfies $a^{\prime \prime}-\kappa a=\kappa|A|, a(0)=a^{\prime}(0)=0$. Now fix a unit vector $u$ and observe that $[(J-A) \cdot u-b]^{\prime \prime} \leq 0$. Since

$$
[(J-A) \cdot u-b](0)=[(J-A) \cdot u-b]^{\prime}(0)=0, \quad(J-A) \cdot u-b \leq 0,
$$

and therefore, $|J-A| \leq b$. It follows that $b^{\prime \prime} \leq \kappa|J| \leq \kappa b+\kappa|A|$. This implies that $\left[(b-a)^{\prime} \hat{\psi}-(b-a) \hat{\psi}^{\prime}\right]^{\prime} \leq 0$. Again, since $(b-a)^{\prime} \hat{\psi}(0)=(b-a) \hat{\psi}^{\prime}(0)=0$, we find that $((b-a) / \hat{\psi})^{\prime} \leq 0$ and therefore, $(b-a) / \hat{\psi} \leq 0$. In particular, $b \leq a$. Finally, we obtain

$$
|J(s)| \leq|J-A|+|A| \leq b+|V| s \leq a+|V| s=|V| \hat{\psi}(s) .
$$

The bound on $\left|J^{\prime}(s)\right|$ is obtained as in Theorem 5.13. Q.E.D.

We also need to know that if $K$ and $V$ vary continuously with respect to a parameter, that the solution $J$ given by Proposition 5.5 varies continuously with respect to the $C^{1}$ topology. Using the integral equation given in the proof to Theorem 5.5, the following is straightforward:

Proposition 5.15. Let $K$ be a continuous matrix valued function on $(0,1) \times$ $[0,1]$ such that $K(\cdot, t)$ satisfies $(5.3)$ for each $t \in[0,1]$. Let $V_{0}, V_{1}:(0,1) \rightarrow$ $\mathbf{R}^{n}$ be continuous and $J_{t}$ the unique solution to $J_{t}^{\prime \prime}+K(\cdot, t) J_{t}=0, J_{t}(1)=$ $V_{0}(t), J_{t}^{\prime}(1)=V_{1}(t)$. Then the map

$$
\begin{aligned}
(0,1) & \rightarrow C^{0}([0,1]) \\
t & \mapsto J_{t}
\end{aligned}
$$

is continuous.

Also, the map

$$
\begin{aligned}
(0,1) & \rightarrow C^{0}([0,1]) \\
t & \mapsto \widehat{J}_{t}
\end{aligned}
$$


where $\widehat{J}_{t}$ is the unique solution to $J_{t}^{\prime \prime}+K(\cdot, t) J_{t}=0, J_{t}(0)=0, J_{t}^{\prime}(0)=V_{1}(t)$ is continuous.

\section{EXISTENCE OF GEODESIC SPRAYS}

Definition. Given an open set $U \subset \widetilde{B}$, a $C^{1}$ map

$$
\Gamma:(0,1] \times U \rightarrow \widetilde{M}
$$

is called a (geodesic) spray if for each $x \in U, \Gamma(\cdot, x)$ is a geodesic ray joining $p$ to $x$ parameterized by a constant times arclength.

The main result of this section is the following:

Proposition 6.1. Given $x \in \widetilde{B}$, there exists a neighborhood $U$ of $x$ and $a$ geodesic spray $\Gamma:[0,1] \times U \rightarrow B$.

Proof. Let $\gamma_{0}:[0,1] \rightarrow B$ be a minimal geodesic ray joining $p$ to $x_{0}$. We want to extend $\gamma_{0}$ to a geodesic spray. Note that the order geodesics in the spray need not be minimal.

Let $\delta=d\left(p, x_{0}\right)$. Let $U=B_{\varepsilon}\left(x_{0}\right)$, where $\varepsilon<\frac{1}{2} \delta$, be a geodesically convex neighborhood of $x$. We construct the spray as a limit of families of geodesics joining each point along $\gamma_{0}$ to $U$.

Given $\sigma \in(0,1]$, let $v_{\sigma}=(1-\sigma) \gamma_{0}^{\prime}(\sigma) \in T_{\gamma_{0}(\sigma)} M$, so that $\exp _{\gamma_{0}(\sigma)} v_{\sigma}=x_{0}$. Let $V_{\sigma} \subset T_{\gamma_{0}(\sigma)} \widetilde{B}$ be the connected component of $\exp _{\gamma_{0}(\sigma)}^{-1} U$ containing $v_{\sigma}$.

Claim. $\exp _{\gamma_{0}(\sigma)}$ is a diffeomorphism of $V_{\sigma}$ onto $U$.

We need to show that given $v \in V_{\sigma}$, a Jacobi field along the geodesic $\gamma(s)=$ $\exp _{\gamma_{0}(\sigma)} s v_{\sigma}, 0 \leq s \leq 1$, can vanish at most once. By the estimates of $\S 5$, it suffices to show that

$$
\int_{0}^{1} s|K(s)| d s<1
$$

where $K(s)$ is the matrix function that appears in the Jacobi equation along $\gamma$. This bound, however, follows easily from the assumed bound on sectional curvature and the following estimate which is also obtained from estimates proved in $\S 5$,

$$
d(p, \gamma(s)) \geq \delta[\sigma+(1-\sigma) s]-\varepsilon \frac{\psi(s)}{\psi(1)} .
$$

We therefore obtain a spray $\Gamma_{\sigma}$ joining $\gamma_{0}(\sigma)$ to $U$.

We now want to show that as $\gamma \rightarrow 0$, the spray $\Gamma_{\sigma}$ converges in the $C^{1}$ norm to a geodesic spray joining $p$ to $U$.

To obtain $C^{0}$ convergence, it suffices to prove that given $0<\sigma, \sigma^{\prime}<1$,

$$
d\left(\Gamma_{\sigma}(s, x), \Gamma_{\sigma^{\prime}}(s, x)\right) \leq c\left|\sigma-\sigma^{\prime}\right|,
$$

where $c$ is independent of $s, x$.

First, consider

$$
d\left(\Gamma_{\sigma}(s, x), \Gamma_{\sigma}(s, x)\right) \leq \int_{\sigma^{\prime}}^{\sigma}\left|\frac{\partial \Gamma_{t}}{\partial t}(s, x)\right| d t \leq \int_{\sigma^{\prime}}^{\sigma}\left|J_{t, x}(s)\right| d t,
$$

where $J_{t, x}$ is the Jacobi field along the geodesic $\Gamma_{t}(\cdot, x)$ satisfying $J_{t, x}(0)=$ $\gamma_{0}^{\prime}(t)$ and $J_{t, x}(1)=0$. Using the assumed bound on sectional curvature, we 
can apply the estimates of $\S 5$ to $J_{t, x}$. In particular, it follows from Lemma 5.11 that $\left|J_{t, x}(s)\right|$ is a decreasing function in $s$. Therefore,

$$
d\left(\Gamma_{\sigma}(s, x), \Gamma_{\sigma^{\prime}}(s, x)\right) \leq \int_{\sigma^{\prime}}^{\sigma}\left|J_{t, x}(1)\right| d t=\int_{\sigma^{\prime}}^{\sigma}\left|\gamma_{0}^{\prime}(t)\right| d t=d\left(p, x_{0}\right)\left|\sigma-\sigma^{\prime}\right| .
$$

Define $\Gamma=\lim _{\sigma \rightarrow 0} \Gamma_{\sigma}$. Since for each $\sigma$ and $x, \Gamma_{\sigma}(\cdot, x)$ is a geodesic, it follows that the limiting curve $\Gamma(\cdot, x)$ is also a geodesic. It only remains to show that $\Gamma$ is a $C^{1}$ family.

First, since $\Gamma$ is a family of geodesics, to show that $\partial \Gamma_{\sigma} / \partial s \rightarrow \partial \Gamma / \partial s$ uniformly on compact subsets of $(0,1] \times U$, it suffices to show that it converges uniformly to $\{1\} \times U$.

Fix $x \in U$ and orthonormal frame $e_{1}, \ldots, e_{n}$ at $x$. Parallel translate the frame along $\gamma_{0}$ and then along the curves given by $\sigma \mapsto \Gamma_{\sigma}(s, x)$. By writing everything in coordinates, it is easily checked that this parallel translation extends to $\gamma$. Now use this frame to expand

$$
\frac{\Gamma_{\sigma}}{\partial s}=T_{\sigma}^{i} e_{i}=T_{1}^{i}-\int_{\sigma}^{1} \frac{\partial T_{\sigma^{\prime}}^{i}}{\partial \sigma^{\prime}} d \sigma^{\prime}
$$

Now observe that

$$
\frac{\partial T_{\sigma^{\prime}}^{i}}{\partial \sigma^{\prime}}=\frac{\partial}{\partial \sigma^{\prime}}\left(e_{i} \cdot \frac{\partial \Gamma_{\sigma^{\prime}}}{\partial s}\right)=e_{i} \cdot \nabla_{\partial / \partial s} J
$$

where $J_{\sigma}=\partial \Gamma_{\sigma} / \partial \sigma$ satisfies

$$
J_{\sigma}^{\prime \prime}+K_{\sigma} J_{\sigma}=0, \quad J_{\sigma}(0)=\gamma_{0}^{\prime}(\sigma), \quad J_{\sigma}(1)=0 .
$$

It follows from Lemma (5.5.11) and Proposition (5.5.15) that $J_{\sigma}$ depends continuously on $\sigma$ and converges in the $C^{1}$ norm as $\sigma \rightarrow 0$. It follows that the integral in (6.2) and therefore $\partial \Gamma_{\sigma} / \partial \sigma$ converges as $\sigma \rightarrow 0$. Observe that all the estimates are uniform, independent of $x \in U$.

Finally, we need to show that $\Gamma$ is $C^{1}$ with respect to $x \in U$. Fix a smooth curve $\mu:[-\delta, \delta] \rightarrow U$ and, abusing notation, denote

$$
\Gamma_{\sigma}(s, t)=\Gamma_{\sigma}(s, \mu(t)) .
$$

Then $J_{\sigma}=\partial \Gamma_{\sigma} / \partial t$ satisfies the Jacobi equation

$$
J_{\sigma}^{\prime \prime}+K_{\sigma} J_{\sigma}=0, \quad J_{\sigma}(0)=0, \quad J_{\sigma}(1)=\mu^{\prime}(t),
$$

where $K_{\sigma}$ is a continuous family of matrix-valued functions satisfying the assumptions used in $\S 5$. Again, this means that $J_{\sigma}$ depends continuously on $\sigma$ and as $\sigma \rightarrow 0, J_{\sigma}$ converges to the corresponding unique solution of the Jacobi equation obtained by setting $\sigma=0$. This completes the proof that $\Gamma$ is a $C^{1}$ family of geodesics. Q.E.D.

The following follows from local regularity properties of Jacobi fields.

Lemma 6.4. Let $\Gamma:[0,1] \times U \rightarrow B$ be a geodesic spray. Then given any smooth curve $\sigma:[0,1] \rightarrow U$, the following partial derivatives are well defined and continuous on $(0,1] \times U$,

$$
\frac{\partial^{2} \Gamma}{\partial s^{2}}, \frac{\partial^{2} \Gamma}{\partial s \partial t}, \frac{\partial^{3} \Gamma}{\partial s^{2} \partial t}=\frac{\partial^{3} \Gamma}{\partial s \partial t \partial s}=\frac{\partial^{3} \Gamma}{\partial t \partial s^{2}} .
$$


Remark. On the other hand, the existence of $\partial^{2} \Gamma / \partial t^{2}$ requires a bound on the covariant derivative of the curvature tensor.

\section{RADIALLY PARALLEL VECTOR FIELDS}

Given a point $p$ in a smooth Riemannian manifold $M$, we say that a vector field on a geodesic ball centered at $p$ is radially parallel if it is parallel along any geodesic passing through $p$. Parallel translation defines a natural 1-1 correspondence between $T_{p} M$ and the set of all radially parallel vector fields. We will construct such vector fields on the image of a spray, even though we do not yet know how to define tangent vectors at the singular point itself.

Definition. Given a spray $\Gamma:[0,1] \times U \rightarrow B$, a $C^{1}$ vector field $V$ on $[0,1] \times U$ is radially parallel if the following hold:

1. For each $x \in U, \Gamma_{*} V$ is parallel along the geodesic ray $\Gamma(\cdot, x)$.

2. Given a smooth vector field $X$ along $U$ and $x \in U$,

$$
\lim _{s \rightarrow 0}\left|\nabla_{\Gamma_{*} X} \Gamma_{*} V(s, x)\right|=0 \text {. }
$$

Proposition 7.1. Let $U \subset \widetilde{B}$ be a simply connected open set. Given a spray $\Gamma:[0,1] \times U \rightarrow B,(s, x) \in[0,1] \times U$, and $v \in T_{(s, x)}[0,1] \times U$, there exists a unique radially parallel vector field $V$ such that $V(s, x)=v$.

Proof. First, define $V$ to be parallel along $\Gamma(\cdot, x)$. Given any other geodesic ray $\Gamma(\cdot, y), y \in U$, we need to extend $V$ to a parallel vector field along $\Gamma(\cdot, y)$.

Let $\sigma:[0,1] \rightarrow U$ be a smooth curve such that $\sigma(0)=x$ and $\sigma(1)=y$. Abusing notation, denote $\Gamma(s, t)=\Gamma(s, \sigma(t))$. For each $\varepsilon \in(1,0]$, let $V_{\varepsilon}$ be the vector field along $\Gamma$ obtained by parallel translating along $\Gamma(\cdot, 0)$, then along $\Gamma(\varepsilon, \cdot)$, and then along $\Gamma(\cdot, t)$. In other words, it is the unique solution to the following set of equations

$$
V_{\varepsilon}(s, 0)=V, \quad \nabla_{\partial / \partial t} V_{\varepsilon}(\varepsilon, t)=0, \quad \nabla_{\partial / \partial s} V_{\varepsilon}(s, t)=0, \quad 0<s, t \leq 1 .
$$

The vector field $V_{\varepsilon}$ should be viewed as an approximation of what we want. We then take a limit of $V_{\varepsilon}$ as $\varepsilon \rightarrow 0$.

To show that the limits exists, let $W(s, t)$ be the unique vector field satisfying

$$
W(s, 0)=V, \quad \nabla_{\partial / \partial t} W(s, t)=0, \quad 0<s, t \leq 1,
$$

and let $e_{1}, \ldots, e_{n}$ be an orthonormal frame along $\Gamma$ satisfying

$$
\nabla_{\partial / \partial s} e_{i}(s, 1)=0, \quad \nabla_{\partial / \partial t} e_{i}(s, t)=0, \quad 0<s, t \leq 1 .
$$

Denoting $v^{i}(s, t)=V(s, t) \cdot e_{i}$, we have

and

$$
\frac{\partial v^{i}}{\partial s}(s, 1)=\nabla_{\partial / \partial s} W \cdot e_{i}
$$

$$
\frac{\partial}{\partial t}\left(\nabla_{\partial / \partial s} W \cdot e_{i}\right)=\nabla_{\partial / \partial t} \nabla_{\partial / \partial s} W \cdot e_{i}=R\left(\frac{\partial}{\partial t}, \frac{\partial}{\partial s}\right) W \cdot e_{i}
$$

Therefore,

$$
\begin{aligned}
v^{i}(0,1) & =v^{i}(1,1)-\int_{0}^{1} \nabla_{\partial / \partial s} W \cdot e_{i} d s \\
& =v^{i}(1,1)-\int_{0}^{1} \int_{0}^{1} R\left(\frac{\partial}{\partial t}, \frac{\partial}{\partial s}\right) W \cdot e_{i} d t d s .
\end{aligned}
$$


The integral is well defined, since

$$
\begin{aligned}
& \left|\int_{0}^{1} \int_{0}^{1} R\left(\frac{\partial}{\partial t}, \frac{\partial}{\partial s}\right) W \cdot e_{i} d t d s\right| \\
& \quad \leq C|V|\left|\int_{0}^{1} \kappa \psi d s\right|=-C|V| \int_{0}^{1} \psi_{0}^{\prime \prime}(s) d s=C|V|\left(\psi^{\prime}(0)-\psi^{\prime}(1)\right) .
\end{aligned}
$$

We then define $V$ along $\Gamma(\cdot, 1)$ to be $v^{i}(0,1) e_{i}$.

Next, we need to show that the definition of $V$ along $\Gamma(\cdot, y)$ is independent of the choice of the curves $\sigma$. Let $\sigma_{0}$ and $\sigma_{1}$ both be curves joining $x$ to $y$. Since $U$ is simply connected, there exists a smooth homotopy $\Sigma:[0,1] \times[0,1] \rightarrow, \Sigma(0,1)=\sigma_{0}(t), \Sigma(1, t)=\sigma_{1}(t)$. Again abusing notation, denote $\Gamma(r, s, t)=\Gamma(s, \Sigma(r, t)), 0<r, s, t \leq 1$. Let $W$ be the unique vector field along $\Gamma$ satisfying $W(r, s, 0)=V(s)$ along $\Gamma(r, s, 0)$ $(=\Gamma(s, x))$ and $\nabla_{\partial / \partial t} W(r, s, r)=0$. For each $r$, the argument above shows that $\lim _{s \rightarrow 0} W(r, s, 1)$ exists. We need to show that the limit is independent of $r$.

Let $e_{1}, \ldots, e_{n}$ be an orthnormal frame that is parallel along $\Gamma(r, s, 0)$ and that satisfies $\nabla_{\partial / \partial t} e_{i}=0$. (Observe that the frame is well defined with respect to $(r, s, t)$, but is not well defined on the manifold itself.) Removing the limit for the moment and mimicking the calculations above, we get

$$
\begin{aligned}
W \cdot e_{i}(1, s, 1) & =W \cdot e_{i}(0, s, 1)+\int_{0}^{1} \nabla_{\partial / \partial r} W \cdot e_{i}(r, s, 1) d r \\
& =W \cdot e_{i}(0, s, 1)+\int_{0}^{1} \int_{0}^{1} R\left(\frac{\partial}{\partial t}, \frac{\partial}{\partial r}\right) W \cdot e_{i}(r, s, t) d r d t .
\end{aligned}
$$

Now let $s \rightarrow 0$. The left-hand side and the first term on the right have welldefined limits by the argument above. Therefore, the integral does, too. On the other hand,

$$
\left|R\left(\frac{\partial}{\partial t}, \frac{\partial}{\partial r}\right) W \cdot e_{i}\right| \leq C|V| \kappa \psi(s)^{2}=-C|V| \psi(s) \psi^{\prime \prime}(s) .
$$

It suffices to show that the lim inf of the right-hand side is 0 . This follows from observing that $\psi(s)=O(s)$ and that $\psi^{\prime \prime}$ is integrable. Finally, it is not difficult to check that the radially parallel vector field constructed is $C^{1}$.

\section{THE LOCAL INVERSE EXPONENTIAL MAP}

Given $x_{0} \in \widetilde{B}$, let $U \in \widetilde{B}$ be a simply connected neighborhood of $x_{0}$ and $\Gamma:(0,1] \rightarrow \widetilde{B}$ a geodesic spray. Fix an orthonormal basis $e_{1}, \ldots, e_{n}$ of radially parallel vector fields on the spray $\Gamma$. We then define the local inverse exponential map to be

$$
\begin{aligned}
E^{-1}: U & \rightarrow \mathbf{R}^{n} \\
x & \mapsto\left(\frac{\partial \Gamma}{\partial s}(s, x) \cdot e_{1}, \ldots, \frac{\partial \Gamma}{\partial s}(s, x) \cdot e_{n}\right) .
\end{aligned}
$$

Observe that $E^{-1}$ is uniquely defined up to a rotation.

Claim. $E^{-1}$ is a $C^{1}$ immersion. 
Proof of claim. The differential of $E^{-1}$ maps a tangent vector $V \in T_{x} U$ to $\left(J^{\prime}(0) \cdot e_{1}, \ldots, J^{\prime}(0) \cdot e_{n}\right)$, where $J$ is the unique Jacobi field along $\Gamma(\cdot, x)$ such that $J(0)=0$ and $J(1)=V$. That the map $J(1) \mapsto J^{\prime}(0)$ is a linear isomorphism follows from Proposition 5.5. The continuity of the map follows from Proposition 5.15.

\section{UNIQUENESS OF GEODESIC SPRAYS}

The idea now is to extend the local inverse exponential map to a globally defined map, $\widetilde{B} \rightarrow \mathbf{R}^{n}$. To do this, we need to show that the map is uniquely determined on a neighborhood of $x_{0} \in \widetilde{B}$ by a geodesic ray $\gamma_{0}$ joining $p$ to $x_{0}$. This follows from the following:

Proposition 9.1. Let $U$ be a connected neighborhood of $x_{0} \in \widetilde{B}$. Any two geodesic sprays $\Gamma_{1}, \Gamma_{2}:[0,1] \times U \rightarrow B$ such that $\Gamma_{1}\left(\cdot, x_{0}\right)=\Gamma_{2}\left(\cdot, x_{0}\right)$ are the same.

Proof. Let $V$ be the set of $x \in U$ such that $\Gamma_{1}(\cdot, x)=\Gamma_{2}(\cdot, x)$. The set $V$ is nonempty by assumption. It is clearly a relatively closed set. We only need to show that it is open, too.

By shrinking $U$, if necessary, we can assume that the local inverse exponential maps defined by the two sprays are both diffeomorphisms. Let $E_{1}, E_{2}$ be the corresponding exponential maps. They can always be defined so that $E_{1}^{-1}\left(x_{0}\right)=E_{2}^{-1}\left(x_{0}\right)$. Let $C_{1}$ and $C_{2}$ be the conic open sets in $\mathbf{R}^{n}$ obtained by joining each point in $E_{1}^{-1}(U)$ and $E_{2}^{-1}(U)$ to the origin by a straight line segment. Let $C=C_{1} \cap C_{2}$. The crucial point here is that with respect to the metric $E_{1}^{*} g$, the only geodesics that start at the origin are exactly the straight line segments. The Jacobi field estimates proved in $\$ 5$ imply that any other geodesic will hit the side of the cone before it reaches the origin. In particular, given any $x$ sufficiently close to $x_{0}$, the curve $E_{1}^{-1}(\Gamma(\cdot, x))$ must lie in $C$ and therefore be one of the straight line segments. In other words, on a sufficiently small neighborhood of $x_{0}$, the sprays $\Gamma_{1}$ and $\Gamma_{2}$ must coincide. Q.E.D.

\section{THE MANIFOLD OF GEODESIC RAYS}

Define $\widehat{S}$ to be the space of geodesic rays staring at $p$. There is a natural map

$$
\widehat{E}: \widehat{S} \times(0, \varepsilon) \rightarrow \widetilde{B} .
$$

Lemma 10.1. $\widehat{S}$ is a connected manifold with a Riemannian metric of constant curvature 1 , and $\widehat{E}$ is a $C^{1}$ diffeomorphism.

Proof. Since every point in $\widetilde{B}$ is joined by a minimal geodesic ray, the map $\widehat{E}$ is onto. We claim that there is exactly one geodesic joining each $x \in \widetilde{B}$ to $p$. Suppose not. Let $\gamma_{0}$ and $\gamma_{1}$ be two geodesic rays ending at $x \in \widetilde{B}$. By "sliding" the endpoint of $\gamma_{0}$ along $\gamma_{1}$, we obtain a geodesic loop with both endpoints at $p$. However, we have assumed that no such geodesic loop exists in $\widetilde{B}$. Therefore, there is exactly one geodesic ray joining $p$ to $x$.

The topology and geometry of $\widehat{S}$ is now determined by the local inverse exponential map. Given a geodesic ray, a neighborhood of the ray corresponds to a geodesic spray. Given an open set $U$ of geodesic rays, a map $U \rightarrow S^{n-1}$ 
is obtained by mapping a geodesic ray to its unit tangent vector represented as a radially parallel vector field. This map is uniquely determined up to an isometry of $S^{n-1}$. Therefore, the pullback of the standard metric of $S^{n-1}$ is globally well defined on $\widehat{S}$. Q.E.D.

Now, since $\widetilde{B}$ is simply connected, so is $\widehat{S}$. Therefore, $\widehat{S}$ is isometric to the standard sphere and we can identify $\widehat{S} \times(0, e) \simeq B(0, \varepsilon) \backslash\{0\} \subset \mathbf{R}^{n}$ using polar coordinates. It is then easy to verify that the metric $\widehat{E}^{*} g$ extends continuously across the origin and that the coordinates given by this map are exactly geodesic normal coordinates of the metric centered at the singularity.

\section{Almost LINEAR AND HARMONIC COORDINATES}

To obtain coordinates in a neighborhood of $p$ for which the metric has better regularity, we turn to the construction of almost linear and harmonic coordinates described in $[9,10]$.

Let $r(x)=d(p, x), x \in B$. We begin with the following observation:

Lemma 11.10. The function $r(x)^{2}$ is $C^{2}$ with respect to $x$.

Proof. Fix a smooth curve $\sigma:[0,1] \rightarrow B$ and, as before, let $\Gamma(s, t)$ be the corresponding family of geodesic rays joining $p$ to $\sigma$. Observe that

$$
|\partial / \partial s|^{2}(s, t)=r(\sigma(t))^{2}
$$

The basic calculation is the following

$$
\frac{1}{2} \frac{\partial\left(r^{2}\right)}{\partial t}=\frac{\partial}{\partial s} \cdot \nabla_{\partial / \partial t} \frac{\partial}{\partial s}=\frac{\partial}{\partial s} \cdot \nabla_{\partial / \partial s} \frac{\partial}{\partial t}=\frac{\partial}{\partial s}\left(\frac{\partial}{\partial s} \cdot \frac{\partial}{\partial t}\right) .
$$

By Lemma 6.4 all of the quantities on the right-hand side are well defined and continuous.

Again, by Lemma 6.4, we can differentiate both sides one more time with respect to $s$, obtaining

$$
\frac{1}{2} \frac{\partial^{2}}{\partial s^{2}}\left(\frac{\partial}{\partial s} \cdot \frac{\partial}{\partial t}\right)=\frac{\partial}{\partial t}\left(\frac{\partial\left(r^{2}\right)}{\partial s}\right)=0
$$

Therefore, since

$$
\left.\frac{\partial}{\partial s} \cdot \frac{\partial}{\partial t}\right|_{s=0}=0
$$

the function $\partial / \partial s \cdot \partial / \partial t$ is linear in $s$ and $\frac{1}{2} \frac{\partial\left(r^{2}\right)}{\partial t}=\left.\left(\frac{\partial}{\partial s} \cdot \frac{\partial}{\partial t}\right)\right|_{s=1}$. Differentiating both sides with respect to $t$ once more, we get

$$
\frac{1}{2} \frac{\partial^{2}\left(r^{2}\right)}{\partial t^{2}}=\left.\frac{\partial}{\partial t}\left(\frac{\partial}{\partial s} \cdot \frac{\partial}{\partial t}\right)\right|_{s=1} .
$$

In particular,

$$
\frac{d r}{d t}=\left.r^{-1} \frac{\partial \Gamma}{\partial s}\right|_{s=1} \cdot \sigma^{\prime}(t)
$$

and

$$
\frac{d^{2} r}{d t^{2}}=r^{-1}\left[\left.\frac{\partial J}{\partial s}\right|_{s=1} \cdot \sigma^{\prime}(t)+\left.\frac{\partial \Gamma}{\partial s}\right|_{s=1} \cdot \nabla_{\sigma^{\prime}(t)} \sigma^{\prime}(t)-\left(\frac{d r}{d t}\right)^{2}\right]
$$


where $J=\partial \Gamma / \partial t$ is a Jacobi field. The right-hand side involves only $\partial \Gamma / \partial s$, $\partial \Gamma / \partial t, \partial^{2} \Gamma / \partial s \partial t$, the metric, and the Levi-Civita connection. Therefore, it is continuous. It follows that $r$ is $C^{2}$ away from $p$. Q.E.D.

Now fix a unit tangent vector $u \in T_{p} B$ and let $\gamma(s)=\exp _{p} s u$. Given $x \in \widetilde{B}$, define

$$
p(x)=d(x, \gamma(r(x))), \quad q(x)=d(x, \gamma(-r(x))),
$$

and

$$
l(x)=4 \frac{q(x)^{2}-p(x)^{2}}{r(x)} .
$$

The function $l(x)$ is called the almost linear function determined by the vector field $u$. At this point, it is straightforward, but somewhat tedious, to check that the estimates and their proofs as given in $[9,10]$ combined with the Jacobi field estimates proved in $\S 5$ extend to prove the following:

Theorem 11.2. Let $p$ be a point singularity of the Riemannian manifold $\widetilde{M}$ such that all the assumptions of Theorem 3.1 are satisfied. Let $l(x)$ be an almost linear function determined by $u \in T_{p} M$. Then $l(x)$ is a $C^{2}$ function on $B(p, \varepsilon)$ satisfying the following estimates:

$$
\begin{aligned}
|l(x)| & =O(r(x)), \\
|\nabla l(x)-u(x)| & =O\left(r(x)^{2}\right), \\
\left|\nabla^{2} l(x)\right| & =O(r(x)),
\end{aligned}
$$

where $u(x)$ is the radially parallel vector field obtained by translating $u$ along geodesic rays.

Now let $e_{1}, \ldots, e_{n}$ be an orthonormal frame of tangent vectors at $p$ and $x^{1}, \ldots, x^{n}$ be the corresponding almost linear functions. The theorem implies that these functions are coordinates on a neighborhood of $p$ and the metric tensor is $C^{1}$ across $p$ with $g_{i j}(0)=\delta_{i j}$ and $d g_{i j}(p)=0$.

Finally, it can be checked that the construction and analysis of harmonic coordinates from almost linear coordinates as described in $[9,10]$ extend to the situation here. This completes the proof of Theorem 3.1.

\section{THE TWO-DIMENSIONAL CASE}

When $N=2$, the argument above fails in the construction of radially parallel vector fields, since $\widetilde{B}$ is not simply connected. We need to show that assumption (ii) of Theorem 3.3 causes the holonomy obstruction to vanish.

Let $\gamma_{0}$ be a geodesic ray and $v$ be a parallel vector field along $\gamma_{0}$. As we go around the singularity and extend $v$ as a radially parallel vector field, we need to check that when we get back to $\gamma_{0}$, the vector field returns to the original value.

Fix $0<p<\varepsilon$. Let $\Gamma:[0,1] \times[0,1] \rightarrow B$ be a $C^{1}$ map such that $\Gamma(1, \cdot):[0,1] \rightarrow B$ parameterizes the level set $r^{-1}(\rho)$ by a constant times arclength and $\Gamma(\cdot, t)$ is the geodesic ray passing through $\Gamma(1, t)$. Observe that $\Gamma(s, 0)=\Gamma(s, 1)$. For convenience let

$$
\sigma=-\left|\frac{\partial \Gamma}{\partial s}\right|^{-1} \frac{\partial \Gamma}{\partial s}, \quad \tau=\left|\frac{\partial \Gamma}{\partial t}\right|^{-1} \frac{\partial \Gamma}{\partial t} .
$$


Observe that $\sigma$ and $\tau$ form an orthonormal frame on $\widetilde{B}$. Let $V$ be a unit vector field that is parallel along $\Gamma(\cdot, 0)$ and $\nabla_{\partial / \partial t} V=0$. We need to show that

$$
\lim _{s \rightarrow 0} V(s, 1)-V(s, 0)=0 .
$$

Define $\theta(s, t)$ to be the angle between $V(s, t)$ and $\tau$, so that

$$
V \cdot \tau=\cos \theta
$$

It suffices to show that

$$
\lim _{s \rightarrow 0} \theta(s, 0)-\theta(s, 1)=2 \pi .
$$

On the other hand, the rate of change of the angle between the tangent and a parallel vector is equal to the curvature of the curve. More precisely, differentiating (12.1) with respect to $t$, we obtain

$$
V \cdot \nabla_{\partial / \partial t} \tau=(\sin \theta) \frac{\partial \theta}{\partial t} .
$$

Now observe that

$$
\nabla_{\partial / \partial t} \tau=\left|\frac{\partial}{\partial t}\right| \nabla_{\tau} \tau=\left|\frac{\partial}{\partial t}\right| k \sigma,
$$

where $k$ is the curvature of the curve $\Gamma(s, \cdot)$. Substituting this into (12.2) leads to $\partial \theta / \partial t=k|\partial / \partial t|$. Therefore,

$$
\begin{aligned}
\theta(s, 1)-\theta(s, 0) & =\int_{0}^{1} \frac{\partial \theta}{\partial t} d t=\int_{0}^{1} k\left|\frac{\partial}{\partial t}\right| d t \\
& =2 \pi+\int_{0}^{s} \int_{0}^{1} K\left|\frac{\partial}{\partial s}\right|\left|\frac{\partial}{\partial t}\right| d s^{\prime} d t
\end{aligned}
$$

by the Gauss-Bonnet theorem, where $K$ is the Gauss curvature. Letting $s \rightarrow 0$, the double integral vanishes. Therefore, $V$ extends to a well-defined radially parallel vector field on $\widetilde{B}$.

The only other point to make is that the manifold $\widehat{S}$ is no longer simply connected. On the other hand, it is a compact 1 -manifold of length $2 \pi$. The rest of the proof as given for $n \geq 3$ also works for $n=2$.

\section{ACKNOWLEDGMENTS}

The first author would like to thank the Max Planck Institute in Bonn, Germany and Rice University in Houston, Texas, for their hospitality. She was partially supported by a Reidler foundation fellowship. The second author would like to thank the Department of Mathematics, University of Colorado in Boulder and the Institut des Hautes Études Scientifiques in Bures-sur-Yvette, France, for their hospitality. The second author was partially supported by an SNF Postdoctoral Fellowship, NSF grant DMS-86-01853, and a Sloan Fellowship.

\section{REFERENCES}

1. M. T. Anderson, Ricci curvature bounds and Einstein metrics on compact manifolds, J. Amer. Math. Soc. 2 (1989), 455-490.

2. A. Besse, Einstein manifolds, Springer-Verlag, 1986. 
3. P. Buser and H. Karcher, Gromov's almost flat manifolds, Astérisque, 81, Soc. Math. de France, Paris, 1981.

4. J. Cheeger and M. Gromov, Collapsing Riemannian manifolds while keeping their curvature bounded. II, preprint.

5. D. DeTurck and J. Kazdan, Some regularity theorems in Riemannian geometry, Ann. Sci. École Norm. Sup. 14 (1981), 249-260.

6. J. Dieudonné, Foundations of modern analysis, Academic Press, 1960.

7. M. Gromov, J. Lafontaine, and P. Pansu, Structures métriques pour les variétés riemanniennes, Cedic, 1981.

8. P. Hartman, Ordinary differential equations, Wiley, 1964.

9. J. Jost, Harmonic maps between Riemannian manifolds, Australian National Univ., 1984.

10. J. Jost and H. Karcher, Geometrische Methoden zur Gewinnung von a-priori-Schranken für harmonische Abbildungen, Manuscripta Math. 40 (1982), 27-77.

11. H. Karcher, Riemannian center of mass and mollifier smoothing, Comm. Pure Appl. Math. 30 (1977), 509-541.

12. D. Yang, Convergence of Riemannian manifolds with integral bounds on curvature. I, preprint, revised January 1990.

Department of Mathematics, Lehigh University, Bethlehem, Pennsylvania 18015

E-mail address: ps02@lehigh.bitnet

Department of Mathematics, Columbia University, New York, New York 10027

E-mail address: yang@d.columbia.edu 